\title{
GRK6 deficiency is associated with enhanced CXCR4- mediated neutrophil chemotaxis in vitro and impaired responsiveness to G-CSF in vivo
}

\author{
Anne Vroon, ${ }^{\star}$ Cobi J. Heijnen, ${ }^{*}, 1$ Roel Raatgever, ${ }^{*}$ Ivo P. Touw, ${ }^{\dagger}$ Rob E. Ploemacher, ${ }^{\dagger}$ \\ Richard T. Premont, ${ }^{\ddagger}$ and Annemieke Kavelaars* \\ *Laboratory for Psychoneuroimmunology, University Medical Center Utrecht, The Netherlands; ${ }^{\dagger}$ Department of \\ Hematology, Erasmus University Medical Center, Rotterdam, The Netherlands; and ${ }^{\ddagger}$ Department of Medicine, \\ Duke University Medical Center, Durham, North Carolina
}

\begin{abstract}
The stromal cell-derived factor-1 (SDF-1)/CXC chemokine receptor 4 (CXCR4) signaling pathway is thought to play an important role in the induction of neutrophil mobilization from the bone marrow in response to granulocyte-colony stimulating factor (G-CSF) treatment. CXCR4 belongs to the family of $G$ protein-coupled receptors. Multiple members of this receptor family are desensitized by agonist-induced $G$ protein-coupled receptor kinase (GRK)-mediated phosphorylation. Here, we demonstrate that in vitro SDF-1-induced chemotaxis of bone marrow-derived neutrophils from GRK6-deficient mice is significantly enhanced and that desensitization of the calcium response to SDF-1 is impaired in GRK6-/- neutrophils. CXCR4 activation by SDF-1 provides a key retention signal for hematopoietic cells in the bone marrow. It is interesting that we observed that in the absence of GRK6, the G-CSF-induced increase in circulating neutrophils is profoundly impaired. Three days after injection of pegylated-G-CSF, significantly lower numbers of circulating neutrophils were observed in GRK6-I- as compared with wild-type (WT) mice. In addition, early/acute neutrophil mobilization in response to G-CSF (3 h after treatment) was also impaired in GRK6-I- mice. However, blood neutrophil levels in untreated GRK6-I- and WT mice were not different. Moreover, the percentage of neutrophils in the bone marrow after G-CSF treatment was increased to the same extent in WT and GRK6-/- mice, indicating that neutrophil production is normal in the absence of GRK6. However, the increased chemotactic sensitivity of GRK6-I- neutrophils to SDF-1 was retained after G-CSF treatment. In view of these data, we suggest that the impaired G-CSFinduced neutrophil mobilization in the absence of GRK6 may be a result of enhanced CXCR4-mediated retention of $P M N$ in the bone marrow. $J$. Leukoc. Biol. 75: 698-704; 2004.
\end{abstract}

Key Words: G protein-coupled receptor $\cdot$ knockout mice $\cdot$ migration $\cdot$ SDF-1 $\cdot$ mobilization

\section{INTRODUCTION}

Chemokines constitute the largest family of cytokines, with over 50 distinct members and at least 16 different receptors known so far. Although originally characterized as inflammatory mediators, it is now becoming clear that the chemokine system regulates multiple physiological and pathological processes, including tumorigenesis, hematopoiesis, and fetal development [1-4]. Stromal cell-derived factor-1 [SDF-1; CXC chemokine ligand 12 (CXCL12)] and its receptor CXC chemokine receptor 4 (CXCR4) have been shown to possess unique properties within the chemokine system. Studies in knockout animals have demonstrated that apart from being a classical inflammatory chemokine, SDF-1 is essential for normal embryogenesis. During ontogeny of the hematopoietic system, SDF-1 appears to play a critical role in inducing the migration of primitive hematopoietic stem and progenitor cells from the fetal liver to the bone marrow [5, 6]. Targeted disruption of SDF-1 or CXCR4 is lethal, and embryos show similar phenotypes in both deficiencies, indicating that SDF-1 interacts exclusively with CXCR4 and vice versa. Absence of CXCR4 or SDF-1 is associated with defective cardiogenesis, lympho- and myelopoiesis, organ vascularization, and development of the cerebellum [6-9].

In adult mice, the CXCR4/SDF-1 chemotactic axis is involved in the regulation of mobilization of hematopoietic progenitor cells and mature neutrophils from the bone marrow in response to granulocyte-colony stimulating factor (G-CSF), which plays a critical role in production and maturation of neutrophils $[10,11]$ but is also known to be a potent stimulus for inducing neutrophil release from the bone marrow [12-14]. The release of immature and mature neutrophils in response to G-CSF from the bone marrow has been associated with the inactivation of the SDF-1/CXCR4 chemotactic pathway [15, 16]. Upon treatment with G-CSF, the level of SDF-1 protein in the bone marrow is significantly decreased $[14,15,17]$. It is interesting that the degree of neutrophil mobilization in re-

\footnotetext{
${ }^{1}$ Correspondence: University Medical Center, Room KC03.068.0, Lundlaan 6, 3584 EA, Utrecht, The Netherlands. E-mail: c.heijnen@wkz.azu.nl

Received July 10, 2003; revised August 12, 2003; accepted October 21, 2003; doi: 10.1189/jlb.0703320.
} 
sponse to G-CSF is correlated with the magnitude of the decrease in bone marrow SDF-1 [14]. In addition, in mice reconstituted with CXCR4-deficient bone marrow cells, premature release of granulocytic precursors was observed [5, 18]. These data suggest that CXCR4 activation by SDF-1 provides a key retention signal for hematopoietic cells in the bone marrow.

CXCR4 belongs to the class of $\mathrm{G}$ protein-coupled receptors (GPCRs). Members of a family of seven GPCR kinases (GRKs) can attenuate the responsiveness of GPCRs [19-21]. Phosphorylation of agonist-activated receptors by GRKs induces a rapid uncoupling of the receptor from $G$ proteins, a process called homologous desensitization. GRK-mediated phosphorylation of the agonist-bound receptor induces uncoupling by promoting the binding of members of the arrestin family to the phosphorylated receptor, which then prevents further $G$ protein activation and enhances receptor internalization [19-21]. The cellular levels of GRKs and arrestins are thought to be very important in this process, as this determines the extent of receptor desensitization and internalization [22, 23].

GRK2, $-3,-5$, and -6 are particularly highly expressed in cells of the immune system [24, 25]. Recently, it was demonstrated that GRK6 is an important regulator of CXCR4 activity in lymphocytes. T cells genetically deficient in GRK6 displayed changes in CXCR4 signaling and SDF-1-induced chemotactic responses [26]. It is interesting that expression levels of GRK6 were shown to be altered during differentiation of the promyelocytic cell line HL-60 toward the neutrophil lineage, suggesting a functional role for this GRK in regulating GPCR responsiveness in neutrophils as well [25].

In this study, we used mice with a targeted deletion of the GRK6 gene to investigate the possible role of GRK6 in regulation of CXCR4-mediated neutrophil chemotaxis in vitro. In addition, we assessed the effect of GRK6 absence on the G-CSF-induced rise in circulating neutrophils in vivo.

\section{MATERIALS AND METHODS}

\section{Mice}

We used GRK6-deficent mice and littermate controls from a mixed C57/Bl6 $\times$ SVJ/129 background [26, 27]. Animals were genotyped by polymerase chain reaction and housed in the Central Animal Facility of Utrecht University (The Netherlands) under specific pathogen-free conditions. The animal committee of the University Medical Center Utrecht approved all animal experiments.

\section{G-CSF}

Pegylated G-CSF (peg-G-CSF), synthesized by coupling recombinant G-CSF $\mathrm{N}$-terminally to $20 \mathrm{kDa}$ polyethylene glycol, was obtained from Amgen (Thousand Oaks, CA). Peg-G-CSF was administered subcutaneously (s.c.) at a dose of $15 \mu \mathrm{g}$ in $150 \mu \mathrm{l}$ phosphate-buffered saline (PBS). At different time points, peripheral blood was obtained via puncture of the orbital plexus or of the heart. Whole blood cell counts were performed using a Bürker counting chamber, and leukocyte differentials were performed on May-Grünwald/Giemsa-stained blood smears.

\section{Isolation of bone marrow cells}

Femurs and tibias were cleaned and subsequently crushed in a mortar. A total bone marrow suspension was obtained and resuspended in RPMI containing $5 \%$ fetal calf serum (FCS). The suspension was filtered and subsequently centrifuged for $5 \mathrm{~min}$ at $1200 \mathrm{rpm}$. Leukocyte differentials of the bone marrow were performed on May-Grünwald/Giemsa-stained cytospins of total bone marrow cell suspensions (in RPMI $+5 \%$ FCS). For chemotaxis assays, erythrocytes and most primitive (blast) cells were removed using Percoll gradients (Amersham Biosciences AB, Uppsala, Sweden). Subsequently, cells were resuspended in RPMI $+0.5 \%$ FCS and were counted.

\section{Chemotactic assay}

Cell migration was assessed using a Transwell system with $5 \mu \mathrm{m}$ pore filters (Costar, Cambridge, MA). Before use, the filters were coated overnight at $4{ }^{\circ} \mathrm{C}$ with $20 \mu \mathrm{g} / \mathrm{ml}$ fibronectin (in PBS). Subsequently, cells were placed in the top well in a total volume of $100 \mu \mathrm{l}$ and $600 \mu \mathrm{l}$ SDF-1 (R\&D Systems, Minneapolis, MN), or medium (RPMI 1640 with $0.5 \%$ FCS) was added to the lower chamber. After $2 \mathrm{~h}$ incubation at $37^{\circ} \mathrm{C}$, cells in the bottom chamber were collected, and the number of migrated PMN was determined by flow cytometry, based on forward- and side-scatter characteristics (FACSCalibur, Becton Dickinson, San Jose, CA).

\section{Calcium reponse}

Bone marrow neutrophils were loaded with Fluo-3 AM (Molecular Probes, Eugene, OR) and stimulated with SDF-1 in RPMI 1640 supplemented with $0.05 \%$ bovine serum albumin (BSA). Changes in mean fluorescence intensity (MFI) were monitored using a FACSCalibur. Traces represent MFI for $200-$ 300 cells/s sampled in 500-ms periods.

\section{Colony assay}

Bone marrow cells were plated in duplicate at densities of $1 \times 10^{4} / \mathrm{ml}$ in methylcellulose medium supplemented with $30 \%$ fetal bovine serum, $1 \%$ BSA $0.1 \mathrm{mM}$ 2-mercaptoethanol, $2 \mathrm{mM} \mathrm{L-glutamine,} \mathrm{and} \mathrm{G-CSF} \mathrm{(100} \mathrm{ng/ml).}$ Colonies (30 cells or more) were counted after 6 days of culture at $37^{\circ} \mathrm{C}$.

\section{Data analysis}

Data are expressed as mean and SEM and were collected in independent experiments using three pairs of animals in each experiment. Wild-type (WT) and knockout samples were analyzed in parallel. Data were analyzed by Student's $t$-test or two-way ANOVA. $P<0.05$ was considered statistically significant.

\section{RESULTS}

\section{Chemotactic responsiveness of neutrophils to SDF-1}

Using a Transwell chemotaxis assay, we assessed the in vitro chemotactic response toward SDF-1 (CXCL12) of bone marrow-derived polymorphonuclear neutrophils (PMN) from untreated WT and GRK6-/- animals. No significant difference was observed in basal motility of PMN (with medium in upper and lower wells) from WT or GRK6 $-/-$ animals (Fig. 1). In addition, the chemokinetic response of WT and GRK6-/PMN to SDF-1 (SDF-1 in upper and lower well) was also similar. However, the chemotactic response of GRK6-/PMN to SDF-1 (with SDF-1 only in the lower well) was significantly enhanced, compared with WT cells.

\section{Desensitization of CXCR4}

GRKs are known to be involved in homologous receptor desensitization of GPCR, the process that prevents a response of these receptors to restimulation with the same agonist. It is known that CXCR4 desensitizes after stimulation with SDF-1 [28]. Moreover, deficient desensitization of GPCR is thought to result in increased functional effects of the agonist. To get more insight about the role of GRK6 in desensitization of CXCR4, 


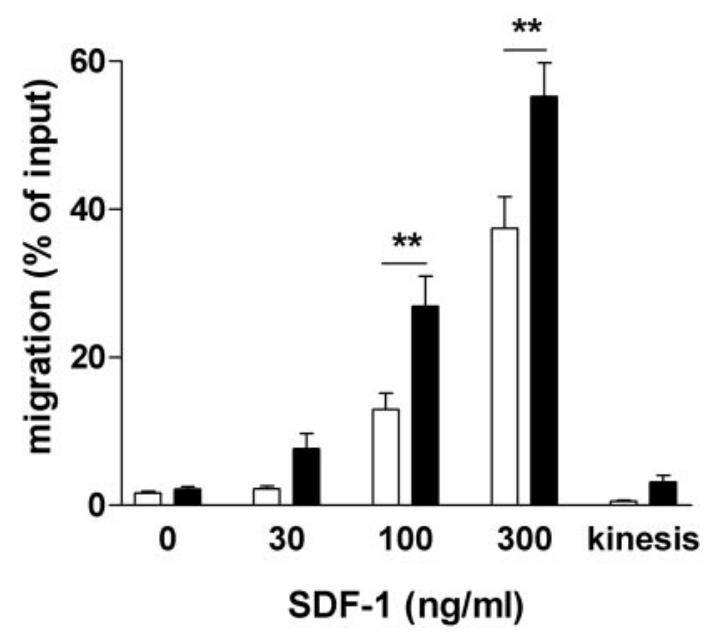

Fig. 1. Chemotactic response of bone marrow-derived PMN from WT and GRK6-/- mice. Open bars, WT; solid bars, GRK6-/- animals. PMN chemotaxis was determined in a Transwell assay. Medium alone or increasing doses of SDF-1 were added to the lower well, and cells were placed in the upper well in medium for $2 \mathrm{~h}$. Spontaneous cellular motility was determined with medium in upper and lower wells. To determine the chemokinetic response (kinesis), $100 \mathrm{ng} / \mathrm{ml} \mathrm{SDF-1} \mathrm{was} \mathrm{added} \mathrm{to} \mathrm{the} \mathrm{upper} \mathrm{and} \mathrm{the} \mathrm{lower}$ wells. Data represent percentage of cells from the total number used that reached the lower well. $\mathrm{n}=5-10$ per group; $* *, P<0.01$ (two-way ANOVA).

we investigated the role of GRK6 in desensitization of the calcium response of neutrophils by pre-exposure of cells to SDF-1. Cells were loaded with Fluo-3 to allow monitoring of changes in intracellular calcium concentrations and stimulated with $100 \mathrm{nM}$ SDF-1. The data in Figure 2 show that the calcium response of GRK6-/- and WT neutrophils is similar. After 5 min stimulation with $100 \mathrm{nM} \mathrm{SDF-1,} \mathrm{cells} \mathrm{were} \mathrm{washed}$

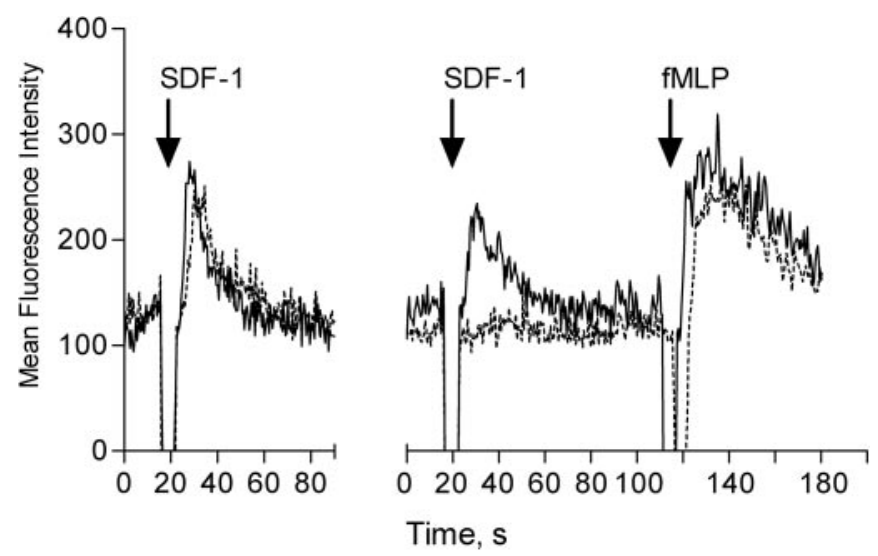

Fig. 2. Desensitization of the calcium response to SDF-1. Bone marrowderived PMN were loaded with Fluo-3, and the change in MFI as an indicator of changes in intracellular calcium concentration was monitored by flow cytometry. Basal Fluo-3 fluorescence was recorded for $15 \mathrm{~s}$, and cells were stimulated with $100 \mathrm{nM} \mathrm{SDF-1.} \mathrm{The} \mathrm{response} \mathrm{to} \mathrm{SDF-1} \mathrm{was} \mathrm{monitored} \mathrm{for} 60 \mathrm{~s}$. Cells were kept in the presence of SDF-1 for $5 \mathrm{~min}$, washed extensively on ice, and resuspended in warm medium, and a second baseline was recorded followed by resimulation with $100 \mathrm{nM}$ SDF-1. As a control, cells were subsequently stimulated with formyl-Met-Leu-Phe (fMLP; $300 \mathrm{nM}$ ). Dotted line, Control; solid line, GRK6-/- PMN. Data are from one representative animal out of three in each group. extensively and restimulated with $100 \mathrm{nM}$ SDF-1. As expected, WT neutrophils do not show an increase in intracellular calcium concentration after restimulation with SDF-1, whereas these cells do respond to stimulation with another stimulus (300 nM fMLP; Fig. 2, dotted line). In contrast, GRK6-/neutrophils that had been prestimulated with $100 \mathrm{nM}$ SDF-1 for $5 \mathrm{~min}$ do respond with an increase in intracellular calcium when they are stimulated a second time (Fig. 2, solid line). These data suggest that desensitization of CXCR4 in neutrophils is impaired in the absence of GRK6.

\section{Peg-G-CSF-induced increase in circulating neutrophils}

SDF-1 and its receptor CXCR4 are involved in the mobilization of bone marrow-derived neutrophils in response to G-CSF. Therefore, we assessed the G-CSF-induced increase in circulating neutrophils in GRK6-/- and WT mice. Blood was drawn at different time points after a single s.c injection of peg-G-CSF, and circulating neutrophil numbers were determined. In WT mice, peg-G-CSF induced a time-dependent increase in the number of circulating PMN, which peaked at day 3 after administration and normalized again at day 5 (Fig. 3A). These data are in line with the results obtained by van Spriel et al. [29] using the same peg-G-CSF. In GRK6-/mice, however, the response to peg-G-CSF was severely impaired. At day 3 after peg-G-CSF injection, PMN levels in peripheral blood from GRK6-/- mice were significantly lower than in WT mice (Fig. 3A). In WT mice, PMN counts were increased approximately sevenfold, whereas the number of blood PMN in GRK6-/- mice had only doubled. Blood neutrophil counts in untreated WT and GRK6-/- animals were similar.
A

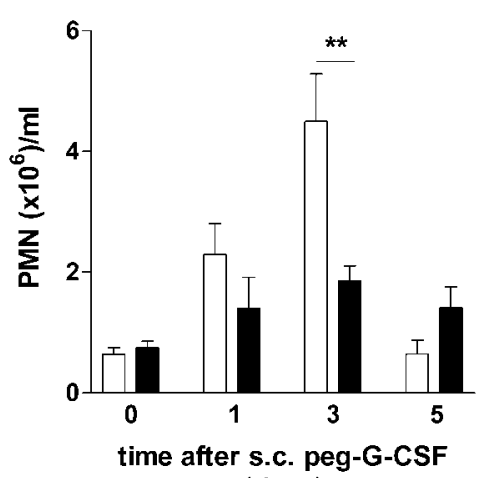

(days)
B

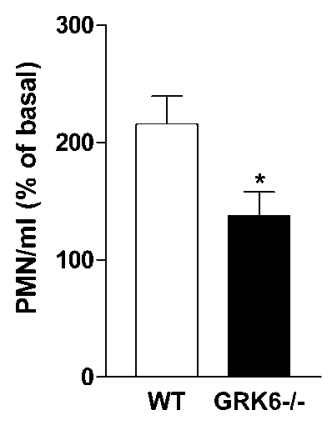

Fig. 3. (A) Neutrophil response to peg-G-CSF treatment. Open bars, WT; solid bars, GRK6-/-. Mice were given a single s.c. dose of peg-G-CSF (15 $\mu \mathrm{g})$, and the number of blood PMN/ml was analyzed at the indicated times. Data represent mean \pm SEM. (B) Short-term neutrophil response to peg-G-CSF. Circulating PMN/ml were determined before and $3 \mathrm{~h}$ after s.c. injection of 15 $\mu \mathrm{g}$ peg-G-CSF. Data are expressed as percentage increase over basal PMN/ml and represent mean \pm SEM. $\mathrm{n}=5-10$ animals per group; $*, P<0.05$; **, $P$ $<0.01$ (Student's $t$-test). 


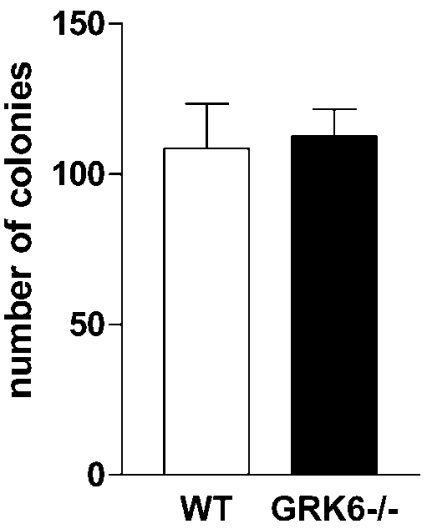

Fig. 4. Colony formation by bone marrow progenitors from WT and GRK6-/- mice. Colonies were grown in the presence of $100 \mathrm{ng} / \mathrm{ml} \mathrm{G-CSF}$. Data represent mean colony numbers + SEM. $\mathrm{n}=5$ per group.

\section{Early neutrophil mobilization induced by peg-G-CSF}

The G-CSF-induced rise in PMN levels at day 3 reflects neutrophil production as well as mobilization. To discriminate between these two processes, circulating neutrophil numbers were assessed $3 \mathrm{~h}$ after s.c. injection of peg-G-CSF (Fig. 3B). As this time period is too short for G-CSF to induce generation of neutrophils, the acute response is thought to primarily reflect neutrophil mobilization from the bone marrow into the blood $[12,30]$. In WT mice, $3 \mathrm{~h}$ after peg-G-CSF treatment, the number of blood PMN was increased by $\sim 115 \%$ over baseline. However, the neutrophil response in GRK6-/- animals was significantly reduced, as compared with WT mice. PMN levels increased by only $\sim 38 \%$ in the GRK6-/- animals, suggesting that G-CSF-induced neutrophil mobilization is impaired in the absence of GRK6 (Fig. 3B).

\section{Cellular composition of the bone marrow before and after GCSF treatment}

The observation that the peg-G-CSF-induced increase in blood PMN levels at day 3 is impaired in the absence of GRK6 might result from impaired mobilization but could also result from reduced neutrophil production or residence in the bone marrow. Therefore, we analyzed the cellular composition of bone marrow from WT and GRK6-/- animals. Under basal conditions, no differences were observed in percentages of immature myeloid cells, eosinophils, monocytes/macrophages, and lymphocytes between WT and GRK6-/- mice (data not shown). In addition, percentages of cells belonging to the granulocytic lineage, such as band neutrophils and polymorphonuclear neutrophils, did not differ between WT and GRK6-/- bone marrow either (data not shown). Thus, the reduced ability of G-CSF to mobilize bone marrow neutrophils does not result from a lower resident neutrophil population in the bone marrow of GRK6-/- animals.

Three days after peg-G-CSF injection, the percentage of neutrophils in the bone marrow had significantly and equally increased in WT and GRK6-/- mice. No differences were observed in the cellular composition of bone marrow from GRK6-/- mice and WT animals, suggesting that the G-CSF- induced production of neutrophils in the bone marrow in GRK6-/- mice is not impaired (data not shown). To further assess the possible role of GRK6 in the G-CSF-induced production of PMN, we determined the in vitro response to G-CSF in colony assays. G-CSF-induced colony formation by bone marrow progenitor cells from WT and GRK6-/- mice was analyzed. As is shown in Figure 4, the number of colonies obtained after stimulation with G-CSF did not differ between GRK6-/- and WT cultures.

\section{Assessment of neutrophil apoptosis}

Increased neutrophil apoptosis might also explain the low numbers of circulating neutrophils in GRK6-/- animals in response to G-CSF. To get more insight into the rate of apoptosis, bone marrow-derived neutrophils were obtained at day 3 after peg-G-CSF injection, and flow cytometric analysis of annexin- $\mathrm{V}$ and propidium iodine-stained samples was performed. As is shown in Figure 5, no difference in the percentage of annexin- $\mathrm{V}^{\text {pos}} /$ propidium iodine ${ }^{\text {neg }}$ cells was observed between GRK6-I- and WT animals. Moreover, when cells were cultured without growth factors for 24 and $48 \mathrm{~h}$, the percentage of annexin-V-positive cells was equally increased in WT and GRK6-/- cell cultures, suggesting that there is no alteration in survival of neutrophils in the absence of GRK6 (Fig. 5).

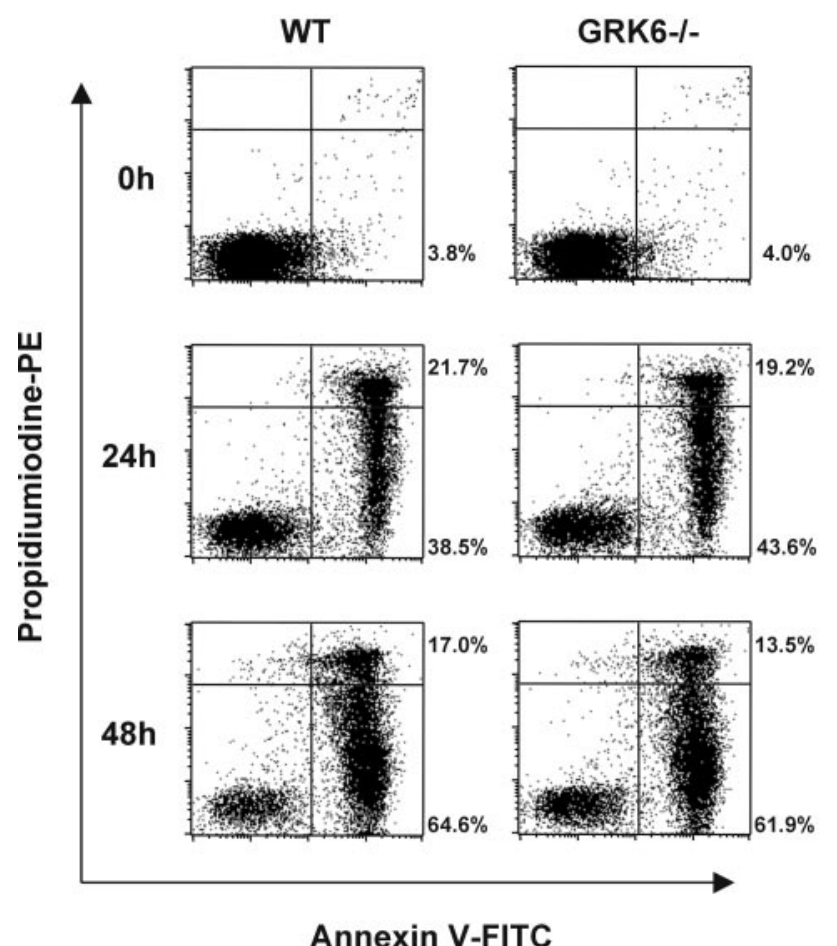

Fig. 5. Assessment of neutrophil apoptosis. Neutrophils were isolated from bone marrow 3 days after injection of peg-G-CSF. Apoptosis was assessed by flow cytometric analysis of cells stained with fluorescein isothiocyanate (FITC)labeled annexin- $\mathrm{V}$ and propidium iodine. In addition, the percentage of apoptotic cells was analyzed after a culture period of 24 or $48 \mathrm{~h}$ without growth factors. Percentage of positive cells is indicated in the dot plot. Four animals per group were analyzed. Shown are representative dot plots of one WT and one GRK6-/- animal. 


\section{Chemotactic responsiveness of neutrophils to SDF-1 after treatment with peg-G-CSF}

To assess whether the difference in chemotactic responsiveness between WT and GRK6-/- PMN is maintained after peg-G-CSF treatment, we also analyzed SDF-1-induced migration of PMN isolated from the bone marrow 3 days after administration of peg-G-CSF. GRK6-/- PMN derived from peg-G-CSF-treated bone marrow showed an increased chemotactic response to SDF-1, as compared with WT cells (Fig. 6). Again, analysis of basal motility as well as the chemokinetic response to SDF-1 revealed no differences between GRK6-/and WT cells (Fig. 6).

\section{DISCUSSION}

In this paper, we demonstrate that the G-CSF-induced increase in circulating neutrophils is significantly impaired in the absence of GRK6. G-CSF has been characterized as the principal hematopoietic cytokine regulating granulopoiesis. In addition, G-CSF is well known for its ability to induce neutrophil release from the bone marrow. Administration of peg-G-CSF induces an immediate neutrophilia, which peaks at $3 \mathrm{~h}$ after injection and results from mobilization of cells from bone marrow to blood. Subsequently, a second increase in blood neutrophils can be observed at day 3 after injection, which is dependent on neutrophil production in the bone marrow. We demonstrate that the early/acute neutrophil response $3 \mathrm{~h}$ after administration of peg-G-CSF is reduced in GRK6-/- animals, suggesting that neutrophil mobilization from the bone marrow is impaired. In addition, peg-G-CSF treatment resulted in little or no increase in circulating neutrophils at day 3 after administration.

The impaired neutrophil response to G-CSF in GRK6-/mice might be the result of altered G-CSF receptor (G-CSFR)

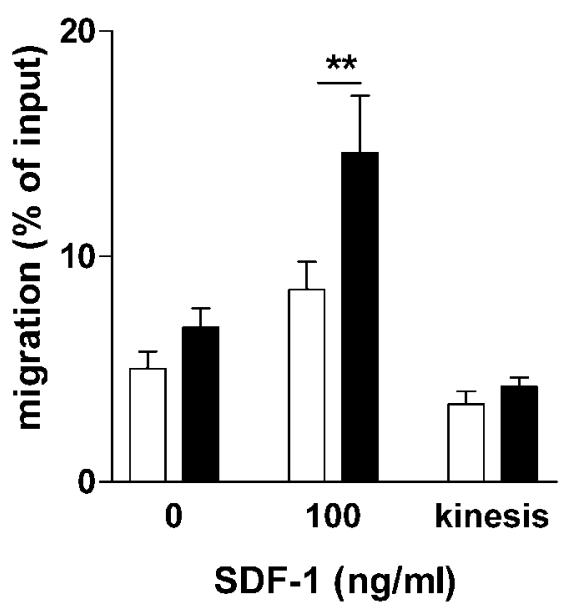

Fig. 6. Motility, SDF-1-induced chemotaxis, and SDF-1-induced chemokinesis (kinesis) of PMN from G-CSF-treated WT and GRK6-/- mice. Bone marrow-derived PMN were obtained 3 days after s.c. injection of $15 \mu \mathrm{g}$ peg-G-CSF. Chemotaxis and chemokinesis were assessed in response to 100 ng/ml SDF-1. Open bars, WT; solid bars, GRK6-/- animals. Data represent percentage of cells from the total number used that reached the lower well. $\mathrm{n}=$ 6 per group; **, $P<0.01$ (two-way ANOVA). signaling in the absence of GRK6. Growth factor receptors such as the G-CSFR do not belong to the GPCR family, which are substrates for desensitization by GRKs. However, previous studies have indicated that GRKs might affect signaling in response to growth factor receptors as well. For instance, overexpression of GRK2 has been shown to affect mitogenic signaling and proliferation of smooth muscle cells and osteoblastic cells in response to platelet-derived growth factor and insulin-like growth factor-1, respectively [31, 32].

G-CSFR signals are essential for proliferation, maturation, and survival of neutrophils in the bone marrow, as well as for the maintenance of homeostatic/steady-state levels of neutrophils in the blood $[11,14,33,34]$. Thus, if G-CSFR signaling is altered in the absence of GRK6, we would expect to observe differences in steady-state blood PMN levels between WT and GRK6-/- animals. However, basal PMN counts were equal in WT and GRK6-/- mice. In addition, analysis of bone marrow cellular composition before and after G-CSF treatment revealed no differences between WT and GRK6-/- animals. Notably, percentages of bone marrow neutrophils had increased equally in both animals. Furthermore, G-CSF-induced colony formation by WT and GRK6-/- bone marrow progenitors did not differ, suggesting that neutrophil production in response to G-CSFR signals is normal. Moreover, there was no difference in percentages of apoptotic bone marrow cells as well as in cell survival between WT and GRK6-/- mice. Finally, Semerad et al. [14] demonstrated that in mixed chimeras reconstituted with WT and G-CSFR mutant bone marrow cells, G-CSFR-deficient and WT neutrophils were mobilized equally by G-CSF [14]. This finding indicates that neutrophil mobilization is not dependent on G-CSFR expression on neutrophils, suggesting that altered signaling of this receptor on GRK6-/- neutrophils is not the cause of the observed, impaired mobilization of these cells in response to G-CSF. Taken together, the data argue against a role of altered G-CSFR signaling in the observed deficient neutrophil response to G-CSF in GRK6-/- animals.

It is thought that G-CSF induces neutrophil mobilization indirectly through the generation of trans-acting signals. The nature of these secondary signals is still largely unknown. However, there is accumulating evidence suggesting that proteolytic disruption of the CXCR4/SDF-1 signaling pathway may represent an important mechanism regulating mobilization of neutrophils in response to G-CSF [14-17]. It is thought that SDF-1/CXCR4 signaling represents a key retention signal for neutrophils/hematopoietic progenitors in the bone marrow and that desensitization of CXCR4 may be an important mechanism in inducing cell mobilization from the bone marrow in response to G-CSF treatment. In line with this hypothesis, we observed an increased sensitivity of bone marrow-derived neutrophils from GRK6-/- animals to migrate toward SDF-1. In naive as well as in G-CSF-treated animals, we demonstrated enhanced SDF-1-induced chemotaxis of GRK6-/- neutrophils as compared with WT cells. Based on these data, we propose that impaired desensitization of CXCR4 in the absence of GRK6 causes the observed, impaired neutrophil mobilization in GRK6-/- mice. Increased sensitivity of the CXCR4/SDF-1 signaling pathway might promote increased retention of neu- 
trophils in the bone marrow, resulting in little or no increase in circulating neutrophils in response to G-CSF.

Our present results, showing that desensitization of the calcium response to stimulation with SDF-1 is impaired in GRK6-/- neutrophils, and the study by Fong et al. [26], showing increased SDF-1-induced GTPase activity in GRK6-/- lymphocytes, are consistent with the hypothesis that GRK6 is required for desensitization of CXCR4, presumably via agonist-induced phosphorylation of the receptor. It is important to realize, however, that CXCR4 has not yet been identified as a substrate for phosphorylation by GRK6.

Surprisingly, in contrast to neutrophils, chemotaxis of T and B cells toward SDF-1 is inhibited in the absence of GRK6 [26], whereas we show here that the chemotactic response of neutrophils toward SDF-1 is increased. We cannot exclude that the difference in chemotactic activity between PMN and T/B cells reflects differences in cell type-specific characteristics of the CXCR4 or the way these distinct cells respond to CXCR4 activation. However, it seems more likely that the differences in SDF-1-induced chemotaxis between PMN and T/B cells are a result of differences in cell type-specific mechanisms of migration that are affected by GRK6. Indeed, it has been suggested that $\mathrm{T}$ cells and PMN use different mechanisms to migrate; T lymphocytes increase locomotion by reducing the duration of migration breaks, and PMN migrate by reducing the frequency of migration breaks [35]. In this respect, it is of interest that we also have data showing increased migration of GRK6-/- PMN toward the chemoattractant leukotriene $B_{4}[50]$.

Retention of neutrophils in the bone marrow is controlled by adhesive interactions involving vascular cell adhesion molecule 1 (VCAM-1), which is expressed by stromal cells, and the integrin very late antigen 4 (VLA-4), which is expressed on neutrophils [36-39]. Inhibition of the interaction between VCAM-1 and VLA-4 by infusion of anti-VCAM-1 or antiVLA-4 was shown to be sufficient to promote mobilization in vivo [37, 40-42]. It is interesting that SDF-1 was observed to stimulate VLA-4-dependent engraftment of immature progenitor cells in nonobese diabetic/severe combined immunodeficiency mice [43] and to promote VLA-4-mediated adhesion of bone marrow cells to fibronectin, VCAM-1, and bone marrow stroma [44], suggesting that CXCR4 signaling is involved in regulation of integrin function. Altered modulation of VLA-4mediated adhesion of neutrophils as a result of altered CXCR4 signaling could thus represent an underlying mechanism for the observed, impaired mobilization of neutrophils in GRK6-/- mice.

Release of neutrophils from the bone marrow in response to G-CSF has been associated with proteolytic degradation of SDF-1 by serine proteases [17], leading to disruption of CXCR4/SDF-1 interactions [45-47]. In addition, these serine proteases were shown to inactivate the VCAM-1 [48, 49]. It can therefore not be excluded that the impaired neutrophil mobilization in response to G-CSF is the consequence of impaired proteolytic degradation of SDF-1 or VCAM-1. However, the possible role of GRK6 in regulation of protease activity is unknown.

In conclusion, we demonstrate that the increase in circulating PMN in response to peg-G-CSF is significantly impaired in the absence of GRK6, suggesting a crucial role for this kinase in regulation of neutrophil mobilization via regulation of responsiveness of GPCRs, which are controlled by GRK6, such as CXCR4.

\section{ACKNOWLEDGMENTS}

The authors thank Prof. Dr. R. J. Lefkowitz (Duke University Medical Center, Durham, NC) for providing the GRK6-/mice. In addition, we thank Mrs. Claudia Heijmans-Antonissen for excellent technical assistance and Mrs. Wil van Dijk-Bosch for her careful evaluation of blood leukocyte differentials.

\section{REFERENCES}

1. Loetscher, P., Clark-Lewis, I. (2001) Agonistic and antagonistic activities of chemokines. J. Leukoc. Biol. 69, 881-884.

2. Mackay, C. R. (2001) Chemokines: immunology's high impact factors Nat. Immunol. 2, 95-101.

3. Rossi, D., Zlotnik, A. (2000) The biology of chemokines and their receptors. Annu. Rev. Immunol. 18, 217-242.

4. Yoshie, O., Imai, T., Nomiyama, H. (2001) Chemokines in immunity. Adv. Immunol. 78, 57-110.

5. Ma, Q., Jones, D., Springer, T. A. (1999) The chemokine receptor CXCR4 is required for the retention of B lineage and granulocytic precursors within the bone marrow microenvironment. Immunity 10, 463-471.

6. Nagasawa, T., Hirota, S., Tachibana, K., Takakura, N., Nishikawa, S., Kitamura, Y., Yoshida, N., Kikutani, H., Kishimoto, T. (1996) Defects of B-cell lymphopoiesis and bone-marrow myelopoiesis in mice lacking the CXC chemokine PBSF/SDF-1. Nature 382, 635-638.

7. Ma, Q., Jones, D., Borghesani, P. R., Segal, R. A., Nagasawa, T., Kishimoto, T., Bronson, R. T., Springer, T. A. (1998) Impaired B-lymphopoiesis, myelopoiesis, and derailed cerebellar neuron migration in CXCR4and SDF-1-deficient mice. Proc. Natl. Acad. Sci. USA 95, 9448-9453.

8. Tachibana, K., Hirota, S., Iizasa, H., Yoshida, H., Kawabata, K., Kataoka, Y., Kitamura, Y., Matsushima, K., Yoshida, N., Nishikawa, S., Kishimoto, T., Nagasawa, T. (1998) The chemokine receptor CXCR4 is essential for vascularization of the gastrointestinal tract. Nature 393, 591-594.

9. Zou, Y. R., Kottmann, A. H., Kuroda, M., Taniuchi, I., Littman, D. R. (1998) Function of the chemokine receptor CXCR4 in haematopoiesis and in cerebellar development. Nature 393, 595-599.

10. Demetri, G. D., Griffin, J. D. (1991) Granulocyte colony-stimulating factor and its receptor. Blood 78, 2791-2808.

11. Liu, F., Wu, H. Y., Wesselschmidt, R., Kornaga, T., Link, D. C. (1996) Impaired production and increased apoptosis of neutrophils in granulocyte colony-stimulating factor receptor-deficient mice. Immunity 5, 491-501.

12. Lord, B. I., Molineux, G., Pojda, Z., Souza, L. M., Mermod, J. J., Dexter, T. M. (1991) Myeloid cell kinetics in mice treated with recombinant interleukin-3, granulocyte colony-stimulating factor (CSF), or granulocytemacrophage CSF in vivo. Blood 77, 2154-2159.

13. Ulich, T. R., del Castillo, J., Souza, L. (1988) Kinetics and mechanisms of recombinant human granulocyte-colony stimulating factor-induced neutrophilia. Am. J. Pathol. 133, 630-638.

14. Semerad, C. L., Liu, F., Gregory, A. D., Stumpf, K., Link, D. C. (2002) G-CSF is an essential regulator of neutrophil trafficking from the bone marrow to the blood. Immunity 17, 413-423.

15. Levesque, J. P., Hendy, J., Takamatsu, Y., Simmons, P. J., Bendall, L. J. (2003) Disruption of the CXCR4/CXCL12 chemotactic interaction during hematopoietic stem cell mobilization induced by GCSF or cyclophosphamide. J. Clin. Invest. 111, 187-196.

16. Shen, H., Cheng, T., Olszak, I., Garcia-Zepeda, E., Lu, Z., Herrmann, S., Fallon, R., Luster, A. D., Scadden, D. T. (2001) CXCR-4 desensitization is associated with tissue localization of hemopoietic progenitor cells. J. Immunol. 166, 5027-5033.

17. Petit, I., Szyper-Kravitz, M., Nagler, A., Lahav, M., Peled, A., Habler, L., Ponomaryov, T., Taichman, R. S., Arenzana-Seisdedos, F., Fujii, N., Sandbank, J., Zipori, D., Lapidot, T. (2002) G-CSF induces stem cell mobilization by decreasing bone marrow SDF-1 and up-regulating CXCR4. Nat. Immunol. 3, 687-694. 
18. Kawabata, K., Ujikawa, M., Egawa, T., Kawamoto, H., Tachibana, K., lizasa, H., Katsura, Y., Kishimoto, T., Nagasawa, T. (1999) A cellautonomous requirement for CXCR4 in long-term lymphoid and myeloid reconstitution. Proc. Natl. Acad. Sci. USA 96, 5663-5667.

19. Lefkowitz, R. J. (1998) G protein-coupled receptors. III. New roles for receptor kinases and $\beta$-arrestins in receptor signaling and desensitization. J. Biol. Chem. 273, 18677-18680.

20. Ferguson, S. S. (2001) Evolving concepts in G protein-coupled receptor endocytosis: the role in receptor desensitization and signaling. Pharmacol. Rev. 53, 1-24.

21. Pitcher, J. A., Freedman, N. J., Lefkowitz, R. J. (1998) G protein-coupled receptor kinases. Annu. Rev. Biochem. 67, 653-692.

22. Schlador, M. L., Nathanson, N. M. (1997) Synergistic regulation of $\mathrm{m} 2$ muscarinic acetylcholine receptor desensitization and sequestration by $\mathrm{G}$ protein-coupled receptor kinase-2 and $\beta$-arrestin-1. J. Biol. Chem. 272, $18882-18890$.

23. Menard, L., Ferguson, S. S., Zhang, J., Lin, F. T., Lefkowitz, R. J., Caron, M. G., Barak, L. S. (1997) Synergistic regulation of $\beta 2$-adrenergic receptor sequestration: intracellular complement of $\beta$-adrenergic receptor kinase and $\beta$-arrestin determine kinetics of internalization. Mol. Pharmacol. 51, $800-808$.

24. Chuang, T. T., Sallese, M., Ambrosini, G., Parruti, G., De Blasi, A. (1992) High expression of $\beta$-adrenergic receptor kinase in peripheral human blood leukocytes. J. Biol. Chem. 267, 6886-6892.

25. Loudon, R. P., Perussia, B., Benovic, J. L. (1996) Differentially regulated expression of the $\mathrm{G}$ protein-coupled receptor kinases, $\beta$ ARK and GRK6, during myelomonocytic cell development in vitro. Blood 88, 4547-4557.

26. Fong, A. M., Premont, R. T., Richardson, R. M., Yu, Y. R., Lefkowitz, R. J., Patel, D. D. (2002) Defective lymphocyte chemotaxis in $\beta$-arrestin2and GRK6-deficient mice. Proc. Natl. Acad. Sci. USA 99, 7478-7483.

27. Gainetdinov, R. R., Bohn, L. M., Sotnikova, T. D., Cyr, M., Laakso, A., Macrae, A. D., Torres, G. E., Kim, K. M., Lefkowitz, R. J., Caron, M. G., Premont, R. T. (2003) Dopaminergic supersensitivity in $\mathrm{G}$ protein-coupled receptor kinase 6-deficient mice. Neuron 38, 291-303.

28. Phillips, R., Ager, A. (2002) Activation of pertussis toxin-sensitive CXCL12 (SDF-1) receptors mediates transendothelial migration of T lymphocytes across lymph node high endothelial cells. Eur. J. Immunol. 32, 837-847.

29. van Spriel, A. B., van den Herik-Oudijk, I., van de Winkel, J. G. (2000) A single injection of polyethylene-glycol granulocyte colony-stimulating factor strongly prolongs survival of mice with systemic candidiasis. Cytokine 12, 666-670.

30. Lord, B. I. (1992) Myeloid cell kinetics in response to haemopoietic growth factors. Baillieres Clin. Haematol. 5, 533-550.

31. Bliziotes, M., Gunness, M., Zhang, X., Nissenson, R., Wiren, K. (2000) Reduced G-protein-coupled-receptor kinase 2 activity results in impairment of osteoblast function. Bone 27, 367-373.

32. Peppel, K., Zhang, L., Huynh, T. T., Huang, X., Jacobson, A., Brian, L., Exum, S. T., Hagen, P. O., Freedman, N. J. (2002) Overexpression of G protein-coupled receptor kinase-2 in smooth muscle cells reduces neointimal hyperplasia. J. Mol. Cell. Cardiol. 34, 1399-1409.

33. Lieschke, G. J., Grail, D., Hodgson, G., Metcalf, D., Stanley, E., Cheers, C., Fowler, K. J., Basu, S., Zhan, Y. F., Dunn, A. R. (1994) Mice lacking granulocyte colony-stimulating factor have chronic neutropenia, granulocyte and macrophage progenitor cell deficiency, and impaired neutrophil mobilization. Blood 84, 1737-1746.

34. Basu, S., Hodgson, G., Katz, M., Dunn, A. R. (2002) Evaluation of role of G-CSF in the production, survival, and release of neutrophils from bone marrow into circulation. Blood 100, 854-861.

35. Entschladen, F., Gunzer, M., Scheuffele, C. M., Niggemann, B., Zanker, K. S. (2000) T lymphocytes and neutrophil granulocytes differ in regulatory signaling and migratory dynamics with regard to spontaneous locomotion and chemotaxis. Cell. Immunol. 199, 104-114.
36. Verfaillie, C. M. (1998) Adhesion receptors as regulators of the hematopoietic process. Blood 92, 2609-2612.

37. Vermeulen, M., Le Pesteur, F., Gagnerault, M. C., Mary, J. Y., Sainteny, F., Lepault, F. (1998) Role of adhesion molecules in the homing and mobilization of murine hematopoietic stem and progenitor cells. Blood $\mathbf{9 2}$, 894-900.

38. Frenette, P. S., Subbarao, S., Mazo, I. B., von Andrian, U. H., Wagner, D. D. (1998) Endothelial selectins and vascular cell adhesion molecule-1 promote hematopoietic progenitor homing to bone marrow. Proc. Natl. Acad. Sci. USA 95, 14423-14428.

39. Liesveld, J. L., Dipersio, J. F., Abboud, C. N. (1994) Integrins and adhesive receptors in normal and leukemic CD34+ progenitor cells: potential regulatory checkpoints for cellular traffic. Leuk. Lymphoma $\mathbf{1 4}$, 19-28.

40. Kikuta, T., Shimazaki, C., Ashihara, E., Sudo, Y., Hirai, H., Sumikuma, T., Yamagata, N., Inaba, T., Fujita, N., Kina, T., Nakagawa, M. (2000) Mobilization of hematopoietic primitive and committed progenitor cells into blood in mice by anti-vascular adhesion molecule-1 antibody alone or in combination with granulocyte colony-stimulating factor. Exp. Hematol. 28, 311-317.

41. Papayannopoulou, T., Nakamoto, B. (1993) Peripheralization of hemopoietic progenitors in primates treated with anti-VLA4 integrin. Proc. Natl. Acad. Sci. USA 90, 9374-9378.

42. Craddock, C. F., Nakamoto, B., Andrews, R. G., Priestley, G. V., Papayannopoulou, T. (1997) Antibodies to VLA4 integrin mobilize long-term repopulating cells and augment cytokine-induced mobilization in primates and mice. Blood 90, 4779-4788.

43. Peled, A., Kollet, O., Ponomaryov, T., Petit, I., Franitza, S., Grabovsky, V., Slav, M. M., Nagler, A., Lider, O., Alon, R., Zipori, D., Lapidot, T. (2000) The chemokine SDF-1 activates the integrins LFA-1, VLA-4, and VLA-5 on immature human CD34 $(+)$ cells: role in transendothelial/stromal migration and engraftment of NOD/SCID mice. Blood 95, 3289-3296.

44. Hidalgo, A., Sanz-Rodriguez, F., Rodriguez-Fernandez, J. L., Albella, B., Blaya, C., Wright, N., Cabanas, C., Prosper, F., Gutierrez-Ramos, J. C., Teixido, J. (2001) Chemokine stromal cell-derived factor-1 $\alpha$ modulates VLA-4 integrin-dependent adhesion to fibronectin and VCAM-1 on bone marrow hematopoietic progenitor cells. Exp. Hematol. 29, 345-355.

45. Delgado, M. B., Clark-Lewis, I., Loetscher, P., Langen, H., Thelen, M., Baggiolini, M., Wolf, M. (2001) Rapid inactivation of stromal cell-derived factor-1 by cathepsin G associated with lymphocytes. Eur. J. Immunol. 31, 699-707.

46. McQuibban, G. A., Butler, G. S., Gong, J. H., Bendall, L., Power, C., Clark-Lewis, I., Overall, C. M. (2001) Matrix metalloproteinase activity inactivates the CXC chemokine stromal cell-derived factor-1. J. Biol. Chem. 276, 43503-43508.

47. Valenzuela-Fernandez, A., Planchenault, T., Baleux, F., Staropoli, I., Le Barillec, K., Leduc, D., Delaunay, T., Lazarini, F., Virelizier, J. L. Chignard, M., Pidard, D., Arenzana-Seisdedos, F. (2002) Leukocyte elastase negatively regulates stromal cell-derived factor-1 (SDF-1)/CXCR4 binding and functions by amino-terminal processing of SDF-1 and CXCR4. J. Biol. Chem. 277, 15677-15689.

48. Levesque, J. P., Takamatsu, Y., Nilsson, S. K., Haylock, D. N., Simmons, P. J. (2001) Vascular cell adhesion molecule-1 (CD106) is cleaved by neutrophil proteases in the bone marrow following hematopoietic progenitor cell mobilization by granulocyte colony-stimulating factor. Blood $\mathbf{9 8}$ 1289-1297.

49. Levesque, J. P., Hendy, J., Takamatsu, Y., Williams, B., Winkler, I. G., Simmons, P. J. (2002) Mobilization by either cyclophosphamide or granulocyte colony-stimulating factor transforms the bone marrow into a highly proteolytic environment. Exp. Hematol. 30, 440-449.

50. Kavelaars, A., Vroon, R., Raatgever, R. P., Fong, A. M., Premont, R. T., Patel, D. D., Lefkowitz, R. J., Heijnen, C. J. (2003) Increased acute inflammation, leukotriene $\mathrm{B}_{4}$-induced chemotaxis, and signaling in mice deficient for G protein-coupled kinase 6. J. Immunol. 171, 6128-6134. 